incurvación, la asociación o no a disfunción eréctil, etc. Si la longitud del pene es normal, con potencia sexual mantenida y la deformidad es leve o moderada $\left(<50^{\circ}\right)$, la técnica de Nesbit o la plicatura de la túnica albugínea son adecuadas para corregir dicha deformidad y proporcional un pene funcional. En cambio, en pacientes con grandes placas, curvaturas graves $\left(>50^{\circ}\right)$, deformidades en reloj de arena, pene corto, flacidez distal o fallo de cirugía previa, estarán indicadas la incisión o la escisión de la placa y la colocación de un injerto, con o sin implantación de prótesis de pene, la cual quedaría reservada para pacientes con disfunción eréctil que no han respondido a las terapias médicas ${ }^{4,5}$. Muchos tejidos de diferente origen han sido usados para corregir el defecto de la túnica albugínea tras la incisión/escisión de una placa de Peyronie, pero el material ideal aún no ha sido encontrado. En la actualidad, los injertos se pueden clasificar en: a) autólogos (fascia lata, fascia temporalis, fascia de Buck, túnica vaginalis, vena safena, dermis, fascia dorsal de los rectos, mucosa bucal, etc.), siendo estos los más utilizados; b) heterólogos (submucosa de intestino delgado porcino, pericardio bovino, etc.), y c) sintéticos (Dacron ${ }^{\circledR}$, Dexon ${ }^{\circledR}$, Gore-tex ${ }^{\circledR}$, etc.), siendo la tendencia actual no utilizar estos últimos a causa de su antigenicidad e inadecuadas propiedades funcionales ${ }^{6,7}$. Las características que debería reunir el injerto ideal son que histológicamente se asemeje a la albugínea, escasa antigenicidad, que sea resistente y a su vez elástico para soportar las erecciones, fácil de obtener y que nos permita obtener injertos de gran tamaño $0^{6}$, ya que es importante que el injerto tenga al menos un 30\% más de superficie que el defecto que se cubrirá, en caso contrario, la retracción del injerto conducirá a la reincurvación postoperatoria. La fascia anterior de los rectos es un tejido que cumple dichas características. Además, la literatura médica publicada hasta el momento, con resultados a largo plazo, han demostrado excelentes resultados clínicos tras la utilización de esta ${ }^{6,8,9}$. En nuestro caso, documentados por las revisiones existentes y dado que necesitábamos un injerto de aproximadamente $5 \times 3 \mathrm{~cm}$, optamos por la fascia anterior de los rectos.

Aunque la mayoría de los autores en la actualidad no recomiendan exéresis completa de la placa sino su incisión, ya que requiere de un parche de gran tamaño, lo cual aumenta el riesgo de retracción, de fallo venooclusivo y de pérdida de la función eréctil, consideramos que en casos seleccionados es una buena opción terapéutica. Respecto a la fascia anterior de los rectos, consideramos que cumple las características de injerto ideal, ya que es un tejido con una morfoestructura similar a la túnica albugínea y nos permite obtener parches amplios, añadiendo escasa morbilidad.

B I B L I O G R A F Í A

1. Tunuguntia HS. Management of Peyronie's disease a review. World J Urol. 2001;19:244-50.

2. Rolle L, Tamagnone A, Bollito E, Ceruti C, Timpano M, Negro CL, et al. Could plaque excision surgery with graft induce a new fibrotic reaction in la Peyronie's disease patients? Arch Ital Urol Androl. 2007;79:167-9.

3. Chilton CP, Castle WM, Westwood CA, Pryor J. Factors associated in the aetiology of Peyronie's disease. Br J Urol. 1982;54:748-50.

4. Kendirci M, Hellstrom WJ. Critical analysis of surgery for Peyronie's disease. Curr Opin Urol. 2004;14:381-8.

5. Levine LA, Lenting EL. A surgical algorithm for treatment of Peyronie's disease. J Urol. 1997;158:2149-52.

6. Craatz S, Spanel-Borowski K, Begemann JF, Olianas R, Fisch M, Hohenfellner R. The dorsal lamina of the rectus sheath: a suitable grafting material for the penile tunica albuginea in Peyronie's disease? BJU Int. 2006;97:134-7.

7. Kadioglu A, Sanil O, Akman T, Ersay A, Guven S, Mammadov F. Graft materials in Peyronie's disease surgery: A comprehensive review. J Sex Med. 2007;4:581-95.

8. Kadioglu A, Sanil O, Akman T, Cakan M, Erol B, Mammadov F. Surgical treatment of Peyronie's disease: A single center experience with 145 patients. Eur Urol. 2008;53:432-9.

9. Pathak AS, Chang JH, Parekh AR, Aboseif SR. Use of rectus fascia graft for corporeal reconstruction during placement of penile implant. Urology. 2005;65:1198-201.

A. Jiménez Pacheco ${ }^{a, *}$, A. López Luque ${ }^{a}$, A. Jiménez Pacheco ${ }^{b}$ y M. Verdú Pérez ${ }^{\mathrm{a}}$

${ }^{a}$ Centro de Trabajo, Servicio de Urología, Hospital Comarcal Santa, Motril, Granada, España

${ }^{\mathrm{b}}$ Servicio de Urgencias, Centro Hospitalario de Alta Resolución

Sierra Segura, Jaén, España

*Autor para correspondencia.

Correo electrónico: anjipa29@hotmail.com

(A. Jiménez Pacheco).

\title{
Prolapso de mucosa uretral en mujer posmenopáusica
}

\section{Urethral prolapse in postmenopausical women}

\section{Sr. Director:}

Los prolapsos uretrales se consideran una patología relativamente infrecuente. Presentan un patrón de edad bimodal, apareciendo en niñas prepuberales y en mujeres postmenopáusicas.

Presentamos el caso de una mujer de 74 años, con antecedentes de HTA y FA en tratamiento con Sintrom ${ }^{\circledR}$. Acude al 


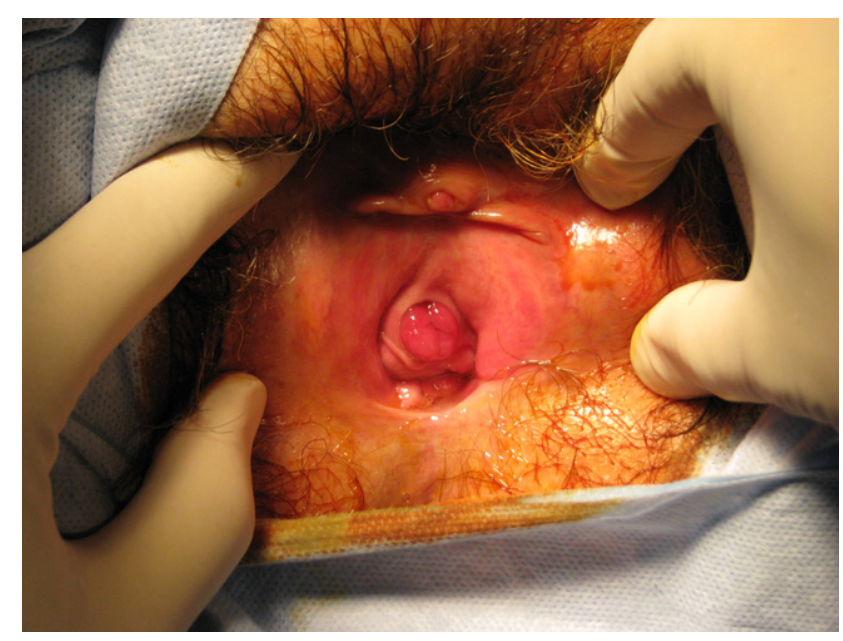

Figura 1 - Mucosa uretral colapsada.

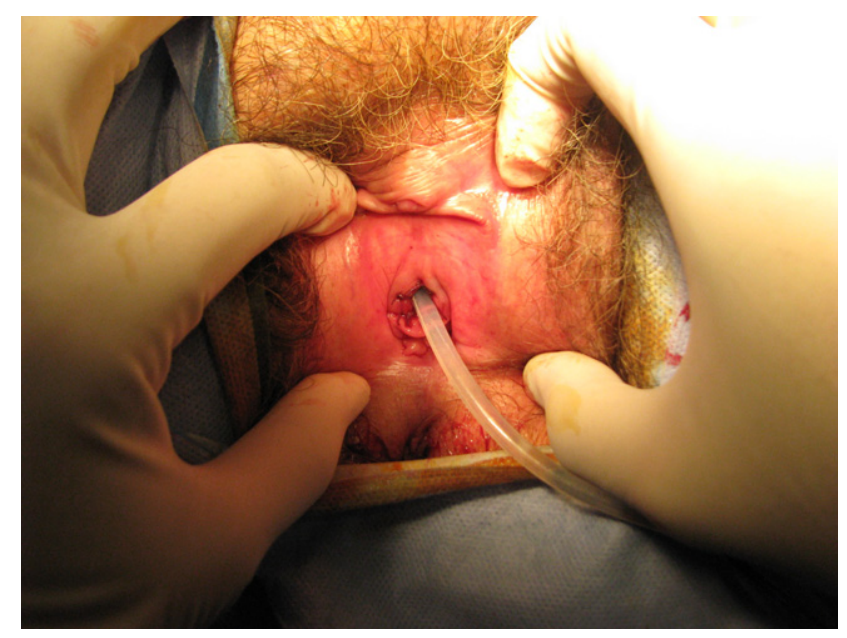

Figura 2 - Meatro uretral tras intervención.

Servicio de Urgencias de nuestro hospital por sangrado genital de varios días de evolución. No presenta clínica miccional. En la exploración se aprecia a nivel de meato uretral un rodete de mucosa prolapsada ( $2 \mathrm{~cm}$ aproximadamente) que sangra con el roce. Tiene molestias leves a la palpación, con posibilidad de reducción de forma parcial con la presión. Con el diagnóstico de prolapso de mucosa uretral, se decide el alta con aplicación tópica de agua oxigenada y crema con corticoides a nivel local. Tras 3 semanas de tratamiento, la paciente presenta mejoría del prolapso, con disminución del tamaño de este y sin sangrado, no obstante, continúa con grandes dificultades para la sedestación, dolor intenso con el roce y rechaza el tratamiento conservador. Se realiza exéresis bajo anestesia local de la mucosa prolapsada (figs. 1 y 2) de forma ambulatoria en régimen de CMA y sondaje vesical durante 3 días. A las tres semanas de la intervención, la paciente se encuentra totalmente asintomática.

El prolapso de la mucosa uretral en la mujer está formado por una eversión circular de la mucosa de la uretra que protruye alrededor del meato. La etiología y la fisiopatología no están claras. La causa podría ser congénita o adquirida, ya que se ha descrito tanto en mujeres ancianas como en niñas prepuberales $^{1-3}$. Los prolapsos congénitos son consecuencia de una mucosa uretral excesivamente redundante por un defecto del tejido colágeno de soporte a nivel de la submucosa. Este hecho produciría una hipermovilidad de la capa mucosa, dando lugar a dicha patología ${ }^{4}$. Los prolapsos adquiridos se han relacionado con múltiples factores desencadenantes tales como aumentos repentinos de presión abdominal $^{11}$ (por pérdidas de adherencia entre la capa de músculo liso longitudinal interna y circular externa), infecciones urinarias recidivantes, traumatismos abdominopélvicos, quemaduras, malnutrición, déficits estrogénicos (mujeres postmenopáusicas, ooforectomía, tras quimioterapia) o abusos sexuales ${ }^{4}$.

La clínica suele ser variable en función de la edad de las pacientes. En niñas prepuberales los síntomas urinarios normalmente están ausentes y suelen ser frecuentes los sangrados vaginales, motivo por el que solicitan asistencia médica. En mujeres postmenopáusicas, en cambio, es mucho más frecuente la disuria, la urgencia y el aumento de frecuencia miccional junto con la posibilidad de retención urinaria $^{1,8}$ (grandes prolapsos que produzcan estrangulación de la mucosa) y dispareunia. En casos de trombosis y necrosis, el sangrado uretral y el dolor local serán los síntomas predominantes ${ }^{9,12}$.

El diagnóstico suele ser clínico, no obstante, deberemos diferenciarlo de patologías como carúnculas uretrales, prolapsos vesicales, divertículos uretrales, prolapso de ureterocele o incluso tumores ${ }^{5,7,10}$.

En la exploración física observaremos una lesión carnosa, discretamente edematosa, con orificio central (a diferencia de la carúncula uretral, en la que el orificio se encuentra en la zona superior). Para diferenciarla del ureterocele prolapsado, algunos autores recomiendan la realización de una cistoscopia ${ }^{4}$. Además es recomendable un análisis anatomopatológico de la mucosa resecada para excluir malignidad.

El tratamiento inicial suele ser conservador, con baños de asiento, cremas de estrógenos y reducción manual ${ }^{6}$. Si estas medidas no son efectivas, o en casos de prolapsos incarcerados o de grandes dimensiones, se recomienda la exéresis quirúrgica de la mucosa prolapsada y la posterior sutura de la mucosa sana al borde uretral, generalmente con buenos resultados postoperatorios. Otras alternativas terapéuticas descritas son la ligadura sobre el catéter uretral, la electrocoagulación y la criocirugía ${ }^{5,11,13}$.

B I B L I O G R A F Í A

1. Sefton E, Shenoy MU. Urethral prolapse in premenarchal girls. BJU Int. 2000;86:402.

2. Templeman C, Hertwek P, Perlman S, Nakajima S. Urethral prolapse in a 7 year old girl. Aust N Z J Obstet Gynaecol. 2000;40:480

3. Valerie E, Gilchrist BF, Frischer J, Scriven R, Klotz DH, Ramenofsky ML. Diagnosis and treatment of urethral prolapse in children. Urology. 1999;54:1082-4.

4. Arango Toro O, Peyri Rey E, Checa Vizcaíno MA. Prolapso de la mucosa uretral. En: Glosa editor. Patología de la uretra femenina y del vestíbulo vaginal. Barcelona: 2004;161-3.

5. Ola B, Arowojolu OA. Urethral prolapse in a west African hospital. Int J Gynaecol Obstet. 1999;66:187-8.

6. Imai A, Horibe S, Tamaya T. Genital bleeding in premenarcheal children. Int J Gynaecol Obstet. 1995;49:41-5. 
7. Rudin JE, Geldt VG, Alecseev EB. Prolapse of urethral mucosa in white female children: Experience with 58 cases. J Pediatr Surg. 1997;32:423-5.

8. Falandry L. Urethral prolapse in young black girls. Report of 12 cases. Prog Urol. 1996;6:392-7.

9. Golomb J, Merimsky E, Braf Z. Strangulated prolapse of the urethra in the elderly female. Int J Gynaecol Obstet. 1985; 23:61-3.

10. Nakao M, Mishina T, Kobayashi T, Maegawi M, Nakagawa S, Imashuku S. A case of rhabdomyosarcoma of the bladder in a child with urethral prolapse. Hinyokika Kiyo. 1983;29: 233-238.

11. Pascual D, Trívez MA, García MA, García de Jalón A, Carela J, Rioja LA. Prolapso uretral tras esfuerzo físico. Corrección quirúrgica. Actas Urol Esp. 2002;26:699-702.
12. Soares MJ, Covita A, Neves T, Monteiro P, Canhoto A, Nogueira R, et al. Prolapso uretral encarcerado. A propósito de un caso. Arch Esp Urol. 2008;61:922-4.

13. Devine PC, Kessel HC. Surgical correction of urethral prolapse. J Urol. 1980;123:856-7.

J.D. Jiménez Parra*, J.L. Cebrián Lostal, F. Lozano Uruñuela y S. Alvarez Bandrés

Servicio de Urología, Hospital Virgen del Camino, Pamplona, España

*Autor para correspondencia.

Correo electrónico: jdjimenez@hotmail.es (J.D. Jiménez Parra).

\section{Una técnica sencilla para el implante testicular con adecuada elección del tamaño: reporte de un caso}

\section{A simple technique for testicular implant insertion and size election: A case report}

\section{Sr. Director:}

La falta de un testículo no sólo puede ocasionar un problema psicológico, sino también físico o estético; es por eso por lo que los pacientes cada vez demandan procedimientos con mejores resultados estéticos, que no solo consisten en reparar la falta, sino en esconder en lo posible las evidencias de la cirugía ${ }^{1}$.

En un estudio sobre pacientes a los que se les practicó una orquidectomía por cáncer testicular, el 27\% de los encuestados que portaban una prótesis testicular estaba disconforme con su posicionamiento, el $37 \%$ no estaba satisfecho con el tamaño y a un tercio de los pacientes no se les ofreció la posibilidad de prótesis ${ }^{2}$.

Presentamos una técnica sencilla para el implante testicular con adecuada elección del tamaño y buen resultado estético así como satisfacción del paciente.

Se presenta el caso de un paciente de 27 años de edad con orquidectomía derecha previa, realizada en su adolescencia debido a la falta de descenso del testículo. Después de recibir antibióticos, se marcó una línea media escrotal así como una incisión supraescrotal. La incisión se infiltró con bupivacaína 0,25 con adrenalina 1:200.000. Se realizó una incisión de $2 \mathrm{~cm}$ supraescrotal y se accedió al escroto mediante disección roma. Para la correcta colocación y elección del tamaño de la prótesis se introdujo una sonda de Foley $16 \mathrm{~F}$ en el escroto (fig. 1). Se fue rellenando el balón de la sonda hasta $32 \mathrm{ml}$, momento en el que el tamaño era similar al testículo contralateral. Una vez que estuvo bien emplazada la sonda y medido el tamaño de la prótesis, se procedió a la expansión tisular inmediata del escroto, rellenando el balón hasta $40 \mathrm{ml}$.
Se sumergió la prótesis en cefazolina y se retiró la sonda después de deshinchar el balón. Los cirujanos procedieron al cambio de guantes y lavado de estos con cefazolina, así como a pincelar la incisión con povidona yodada. Se insertó la prótesis, que fue colocada y fijada a la parte baja del escroto

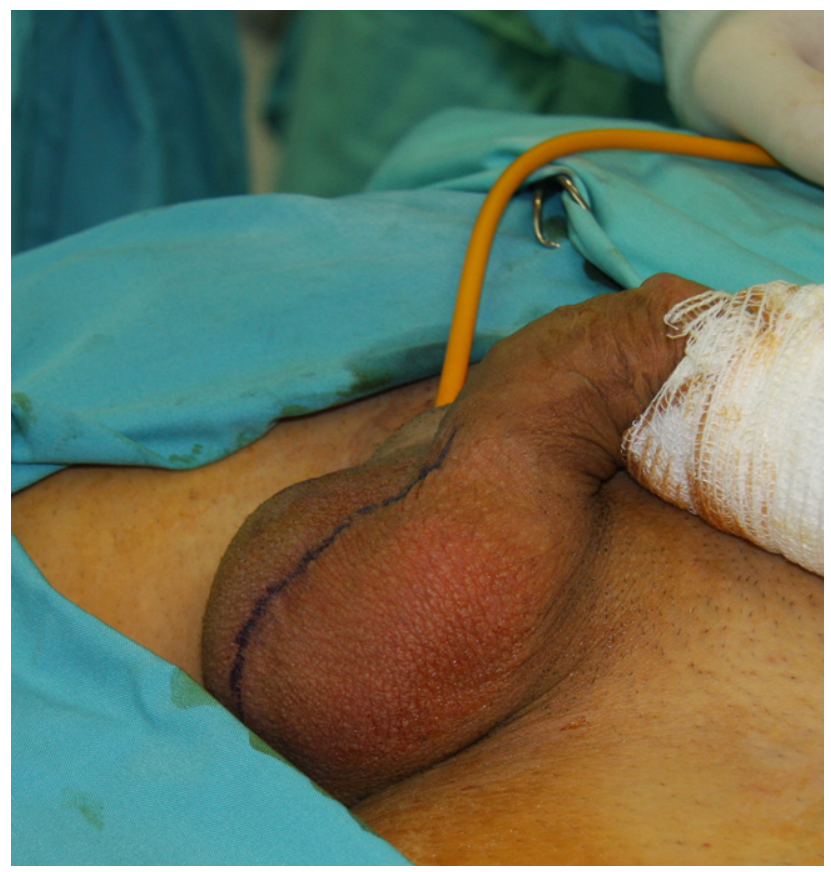

Figura 1 - La inserción en el escroto de un catéter de Foley 16F fue usada para el correcto emplazamiento y medición del tamaño de la prótesis. 